\title{
Matériaux de friction : formulation simplifiée pour la compréhension du rôle des constituants et de l'impact du procédé d'élaboration
}

\author{
Anne-Lise Cristol ${ }^{1,2,3}$, Mouna Baklouti ${ }^{1,2,3,4}$, Nesrine Hentati ${ }^{1,2,3,4}$, Yannick Desplanques ${ }^{1,2,3}$, \\ Riadh Elleuch ${ }^{1}$, Denis Najjar ${ }^{1,2,3}$ et Mohamed Kchaou ${ }^{1}$ \\ ${ }^{1}$ Univ Lille Nord de France, 59000 Lille, France \\ ${ }^{2}$ ECLille, LML, 59650 Villeneuve d'Ascq, France \\ ${ }^{3}$ CNRS, UMR 8107, 59650 Villeneuve d'Ascq, France \\ ${ }^{4}$ Université de Sfax, Ecole Nationale d'Ingénieurs de Sfax, LASEM, 3038 Sfax, Tunisie
}

\section{INTRODUCTION}

Les matériaux de friction sont des systèmes complexes de par leur formulation et leur procédé de fabrication. Leur formulation peut renfermer jusque 40 constituants introduits de manière empirique sans réelle compréhension de leur rôle ni des synergies entre constituants. Leur formulation comprend une succession d'étape dont l'impact sur le matériau et sur son comportement en frottement est mal maîtrisé. Malgré les évolutions constantes des normes environnementales conduisent à des modifications parfois profondes des matériaux, ces derniers doivent maintenir leur performance et garantir leur rôle sécuritaire. Afin de maintenir cet objectif, il est primordial de comprendre les liens entre formulation, procédé et comportement du matériau. Dans cet objectif, le développement d'une formulation simplifiée est proposé : le nombre de ces constituants est réduit et son procédé d'élaboration connu et maîtrisé. Il s'agit ensuite d'utiliser le matériau de friction à formulation simplifiée pour l'étude du rôle des constituants et de l'impact du procédé de fabrication sur les propriétés des matériaux et leur performance en frottement et usure.

\section{MATÉRIAU À FORMULATION SIMPLIFIÉE}

Le matériau à formulation simplifiée est un matériau déviré d'une formulation industrielle contenant 13 constituants. Suivant le rôle qu'ils jouent sur les propriétés du matériau ou sur le comportement en frottement, les constituants peuvent être rangés par classe : liant, fibre de renfort, lubrifiant, abrasif, modificateur de frottement et remplisseur.

Les constituants conservés dans la formulation simplifiée sont ceux qui sont majoritaires dans chacune des classes, ils sont alors introduits avec le pourcentage total de la classe considérée (Tableau 1). Par exemple, des trois remplisseurs présents dans la garniture industrielle (baryte, carbonate de calcium et craie), seule la baryte est conservée à hauteur de $45 \%$ représentant le total des 3 remplisseurs de la formulation industrielle. Le procédé de fabrication est composé des étapes suivantes : mélangeage,
Tableau 1. Composition de la formulation simplifiée.

\begin{tabular}{llc}
\hline Classification & Constituants & \%massique \\
\hline Liant & Résine phénolique & 14 \\
Fibre de renfort & Fibre minérale & 22 \\
Lubrifiant & Graphite & 10 \\
Abrasif & Alumine & 2 \\
Modificateur de & Caoutchouc & 7 \\
frottement & & \\
Remplisseur & Baryte & 45 \\
\hline
\end{tabular}

préformage à froid, moulage à chaud sous pression et postcuisson.

Le matériau à formulation simplifiée peut alors être modifié pour étudier le rôle d'un constituant ou d'une étape du procédé. Dans le cas de l'étude du rôle du laiton, la formulation simplifiée (notée FS) est modifiée par l'ajout de $6 \%$ de laiton compensé par une diminution du pourcentage de baryte (formulation notée FSL). Dans le cas de l'étude de l'impact de la température et de la durée de moulage à chaud, le matériau à formulation simplifiée est élaboré en conservant un moulage à $140^{\circ} \mathrm{C}$ pendant 18 min (matériau noté 14018) et un second matériau est fabriqué en modifiant le couple de paramètres pour mouler à $160^{\circ} \mathrm{C}$ pendant 9 min (matériau noté 16009).

\section{CARACTÉRISATION MATÉRIAU}

La microstructure du matériau est caractérisée par des observations en microscopie optique et en microscopie électronique à balayage. Les observations sont réalisées dans la direction d'application de la charge lors du moulage à chaud ou perpendiculairement à celle-ci. En effet, la microstructure présente une anisotropie puisque les particules longues (i.e. les copeaux de laiton Figure 1a) s'alignent dans perpendiculairement à la direction d'application de la charge comme présenté Figure $1 \mathrm{~b}$ sur une observation d'un copeau de laiton dans le sens orthogonale à la direction de moulage.

Cette anisotropie se retrouve dans les mesures de propriétés effectuées sur le matériau à formulation simplifiée et sur le matériau à formulation modifiée 
a)
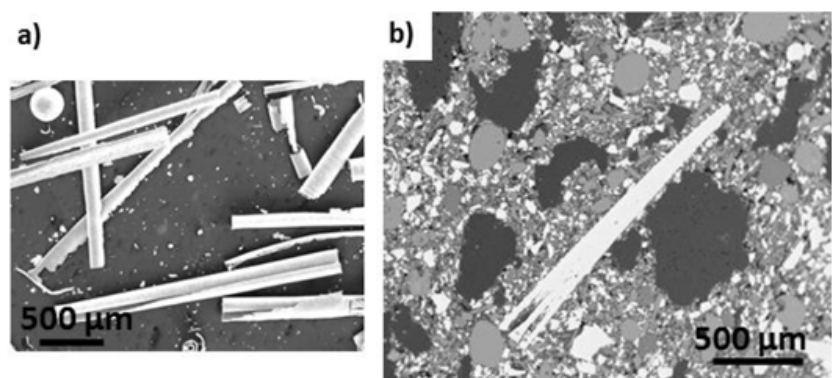

Figure 1. Observation MEB des copeaux de laiton : a) avant introduction dans le mélange, b) dans le matériau.

Tableau 2. Propriétés des matériaux à formulation simplifiée et modifiée avec du laiton.

\begin{tabular}{|c|c|c|}
\hline Propriété & $\begin{array}{l}\text { Matériau à } \\
\text { formulation } \\
\text { simplifiée }\end{array}$ & $\begin{array}{c}\text { Matériau } \\
\text { avec laiton }\end{array}$ \\
\hline Densité $\left(\mathrm{g} . c m^{-1}\right)$ & 2,13 & 2,17 \\
\hline $\begin{array}{l}\left.\text { Conductivité thermique. } \mathrm{K}^{-1}\right) \\
\left(\mathrm{W} \cdot \mathrm{m}^{-1} \cdot \mathrm{K}^{-1}\right)\end{array}$ & 1,04 & 1,31 \\
\hline $\begin{array}{l}\text { Dilatation thermique } \\
\text { sens normal }(\%)\end{array}$ & 0,34 & 0,33 \\
\hline $\begin{array}{l}\text { Dilatation thermique } \\
\text { sens tangentiel }(\%)\end{array}$ & 0,34 & 0,25 \\
\hline Module en compression & 4,65 & 5,27 \\
\hline sens tangentiel (GPa) & 5,39 & 5,35 \\
\hline
\end{tabular}

avec laiton (Tableau 2). L'ajout de laiton conduit à une augmentation de la densité et de la conductivité thermique de par les propriétés du laiton par rapport à la baryte qu'il remplace. L'anisotropie induite par l'introduction des copeaux de laiton est mise ne évidence par des mesures de dilatation thermique et de module de compression dans le sens normal et dans le sens tangentiel : l'ajout de laiton diminue la dilatation thermique dans le sens tangentiel et accroît le module de compression dans le sens normal. Ces caractérisations sont cependant rendues difficiles par la forte hétérogénéité du matériau conduisant à un volume élémentaire représentatif important pas toujours compatible avec les tailles d'échantillons requises pour les diverses techniques mises en œuvre.

\section{COMPORTEMENT EN FROTTEMENT ET USURE}

Le comportement en frottement et usure est étudié par le biais d'essais de frottement discontinu dans différentes gammes de température. Les essais sont réalisés à pression et vitesse constantes et sont pilotés en température. Le contact est maintenu en position On jusqu'à atteindre la température maximale souhaitée puis il est basculé en position Off pour permettre un refroidissement du système. 30 cycles On/Off sont réalisés dans la gamme de température visée.

Dans le cadre de l'étude des paramètres de moulage à chaud, les deux matériaux étudiés (14018 et 16009) présentent les mêmes niveaux de coefficient de frottement dans les trois gammes de températures testées. Par contre,

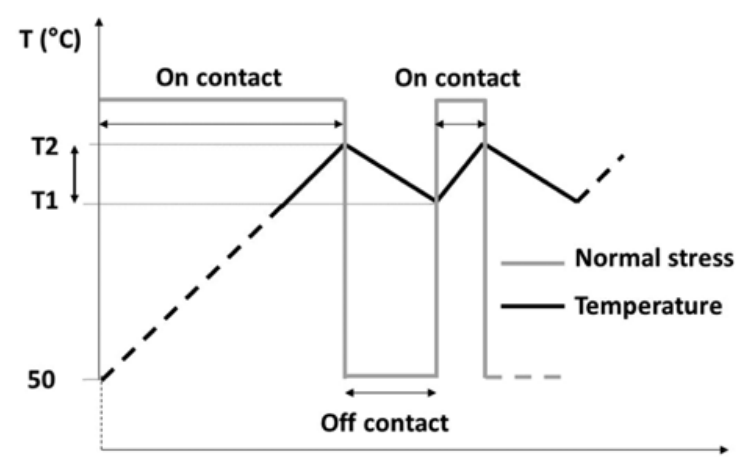

$t(s)$

Figure 2. Séquence d'essais de frottement.

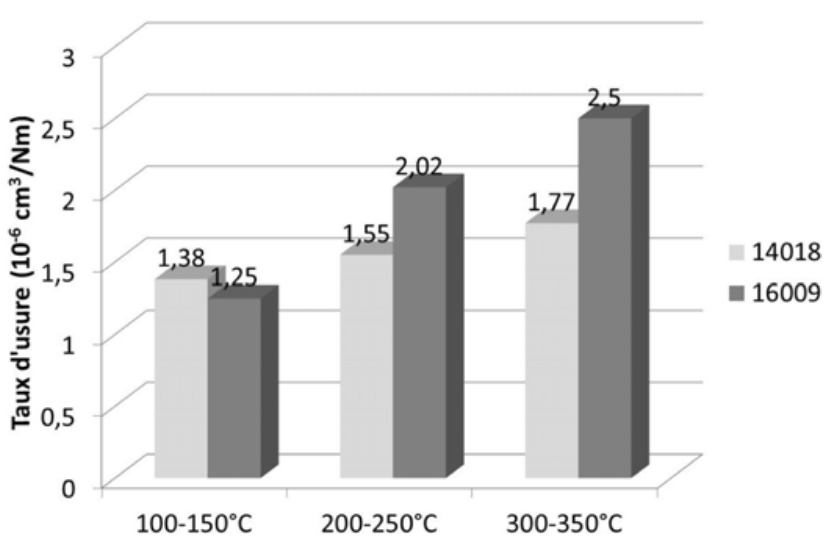

Figure 3. Taux d'usure des matériaux 14018 et 16009 dans les différentes gammes de température testées.

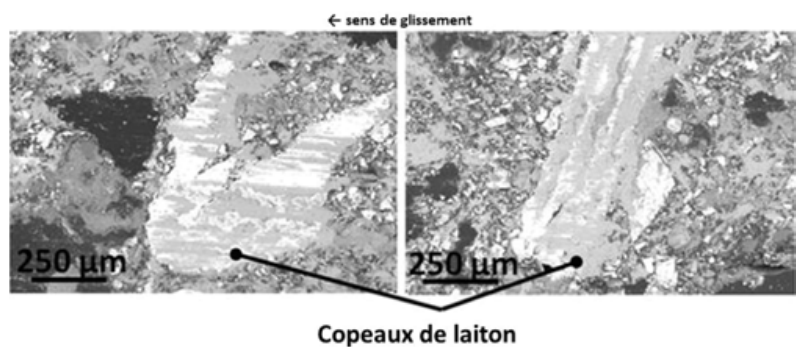

Figure 4. Observations MEB des copeaux de laiton.

le taux d'usure des matériaux est similaire dans la gamme $100-150^{\circ} \mathrm{C}$ mais le matériau 16009 présente un taux d'usure plus élevé que le matériau 14018 dans les gammes $200-250^{\circ} \mathrm{C}$ et $300-350^{\circ} \mathrm{C}$ (Fig. 3).

\section{MÉCANISMES DE FROTTEMENT ET D'USURE}

Après essais de frottement, les surfaces des matériaux de friction sont observées afin d'identifier les mécanismes de frottement et d'usure mis en jeu. La Figure 4 présente des observations MEB de copeaux de laiton présents dans la formulation FL. Les copeaux de laiton jouent le rôle de plateaux primaires dans le contact en assurant la portance. En amont de ces copeaux, le $3^{\text {ème }}$ corps sous forme de poudre s'accumule puis se compacte jusqu'à former des 
plaques planes qui participent elles aussi à la portance sous la forme de plateaux secondaires.

\section{CONCLUSION}

Le développement d'un matériau à formulation simplifiée a servi de base à l'étude de matériaux modifiés en composition ou en procédé d'élaboration afin de comprendre les liens entre formulation/procédé, propriétés matériaux et performance en frottement et usure. Il apparait cependant que la principale difficulté rencontrée est due à la forte hétérogénéité du matériau qui conduit à un volume élémentaire représentatif de taille importante. Il s'agit de simplifier le matériau en limitant la taille et la distribution de taille des constituants puis d'effectuer une caractérisation microstructurale de l'hétérogénéité en 3D via des observations en tomographie. 